\title{
New-age Supercomputers: Hi-Speed Networks and Information Security
}

\author{
Andrey Molyakov \\ Institute of Information Technologies and Cybersecurity, Russian State University for the Humanities, Moscow, Russia \\ Email address: \\ andrei_molyakov@mail.ru \\ To cite this article: \\ Andrey Molyakov. New-age Supercomputers: Hi-Speed Networks and Information Security. Journal of Electrical and Electronic \\ Engineering. Special Issue: Science Innovation. Vol. 7, No. 3, 2019, pp. 82-86. doi: 10.11648/j.jeee.20190703.12
}

Received: August 18, 2019; Accepted: September 21, 2019; Published: October 9, 2019

\begin{abstract}
The author describes computing strategic tasks that are used for ensuring defense and national security, the most important scientific, technical, biomedical and sociology tasks. Most typically, these are capability-based tasks. Supercomputers for their solution are respectively called Technical Capability, i.e. machines of extreme technical capabilities. Machines of this segment are also called High End Computers (HEC), and in our terminology - strategic supercomputers (SCs). Moving to the engineering level, author says that for tasks with good spatio-temporal work with memory, cache memory and schemes for automatically pre-loading data into the cache memory can be effectively used. This can significantly reduce the average memory access time of several hundred processor cycles to fractions of a processor cycle. Such tasks are usually called computational or cache-friendly (cach-friendly) - CF tasks. On tasks with poor spatio-temporal work with memory, the cache memory is useless, so each memory access is hundreds of processor cycles, the processor is idle because of this, and therefore the real performance is in units or even a fraction of a percent of the peak. Such tasks are historically called tasks with intensive irregular work with memory - Data intensive tasks (DIS-tasks). The given examples of spatially-temporal work with task memory and real characteristics of equipment operation in such different modes are given in order to illustrate that in practice different types of supercomputers are needed, for example, for CF- and DIS-tasks.
\end{abstract}

Keywords: Virtualization, Information Security, Supercomputers, Security Descriptor

\section{Introduction}

The simplest and most commonly used definition of a supercomputer is this: a supercomputer is a computing device that outperforms a personal computer by at least one or two orders of magnitude. This definition is controversial, because in addition to performance, supercomputers also have a number of characteristics.

A more correct definition, in our opinion, is this: a supercomputer is a computer system that has orders of magnitude greater performance in a number of the following six main characteristics: - performance on arithmetic operations on floating point numbers;

1. The amount of RAM;

2. Memory bandwidth;

3. Delay in performing operations with memory;

4. Bandwidth of the communication network;

5. Delay in the operation with the communication network.
These characteristics of supercomputers are tens, hundreds and thousands of times higher than the corresponding characteristics of personal computers and servers. In this case, it is necessary to distinguish between peak and real characteristics that the equipment can provide. The level of proximity of real characteristics obtained in solving problems to the peak characteristics of equipment is the most important indicator of the quality of the architecture of a supercomputer and its software, the quality of the application program $[1,2]$.

\section{Methods and Technologies of Creating New-aged Supercomputer Centers}

Historically, supercomputers, nevertheless, distinguished by the total power indicator (the totality of the listed characteristics) into the following four segments: lower segment - level of use in the laboratory / sector; middle segment - department / management level of a large 
enterprise; high segment - the level of a large enterprise; the highest (strategic) segment is the level of a large state laboratory or public service.

The enterprises in which supercomputers of the highest level segment are in demand are nuclear laboratories (Los Alamos, Livermore, Arzamas, Snezhinsk), computer centers of intelligence services (NSA, CIA), state troop control systems (General Staffs and General Staffs of the armed forces). Supercomputers of the highest segment are focused on solving strategic problems, can provide simultaneous solution of a huge number of tasks from small to large complexity (processing such as capacity-based, calculating the total large volume), as well as one or more ultra-large tasks (processing capability-based, at the limit of opportunity, surpassing any technique that can be created). The tasks of the higher segment are usually called strategic, they can be associated with calculations, as well as working with information, which usually requires extreme capabilities for processing information flows and the amount of RAM and external memory used. Strategic tasks are usually the tasks of ensuring defense and national security, the most important scientific, technical, biomedical and sociology tasks. Most typically, these are capability-based tasks. Supercomputers for their solution are respectively called Technical Capability, i.e. machines of extreme technical capabilities. Machines of this segment are also called High End Computers (HEC), and in our terminology - strategic supercomputers, or abbreviated SCS Some of the most powerful models of SCSN are called ultracomputers; they are used to solve the single, most important state tasks. For example, in the United States an annual list of approximately ten such tasks is established, which is approved by the President [3].

Of interest is the relationship between the types of problems being solved and the characteristics of the supercomputers mentioned above. Intelligence tasks are the most demanding on the quality of the memory subsystem and the communication network. In these tasks, the level of real productivity may be less than $1 \%$. According to these indicators, the tasks of monitoring the readiness of nuclear weapons are in second place (in terms of applied importance, they are still in first place), and according to American data, the level of real productivity in these tasks is $1-12 \%$ of peak productivity.

Currently, the field of application of supercomputer technology in the United States and a number of leading foreign countries has expanded significantly. Supercomputer computing began to play a central role in modern science and technology, something that had never happened before. The tasks of the intelligence communities have now gained importance.

In addition, supercomputers suitable for these tasks, but in the on-board version, will be used for the outgoing military tasks of automated intelligent control of weapon systems and reconnaissance and strike groups of troops, tasks of creating autonomous combat robots, which is generally associated with modern views on wars new, sixth generation [4].
Recent US studies to identify the main areas of use of supercomputers allow us to distinguish the following areas (taking into account priority), although the composition and priorities of applications in national programs of other states are somewhat different.

1. Monitoring the combat readiness of nuclear arsenals and the development of a new generation of nuclear weapons. It is important to note that the average time to solve one problem of this type is about a year, and the real efficiency of using equipment, real productivity, is only 1 to 12 percent of the peak.

2. Electronic intelligence and other tasks of intelligence communities. The most difficult are the tasks of radio intelligence related to cryptanalysis and understanding of data from messages, the difficulties of solving these tasks also impede the fight against terrorism.

The tasks of understanding data are either not being solved in the proper amount at all, or with a real productivity of 0.1 1 percent.

3. Military tasks - from the tasks of the optimal conduct of military operations to the scientific and technical tasks of developing modern weapons.

1) Operational prediction of the state of the ocean and weather;

2) Prediction of the spread of impurities in air and water;

3) Engineering design of aircraft, ships and others samples of military equipment;

4) Study of methods for the effective use of weapons;

5) Cryptanalysis;

6) Ensuring survivability and stealth technology;

7) Operational collection and processing of intelligence information, surveillance, aerospace reconnaissance and target designation;

8) Processing signals and images using new methods of intelligent processing, including the use of electronicdigital cards of various types;

9) Missile defense and protection of space objects;

10)Testing and evaluation of the created control systems of different levels.

4. Prediction of climate.

5. Plasma physics - solving the problems of creating thermonuclear reactors.

6. The solution of engineering problems of creating transport - cars, aircraft, ships, spaceships.

7. Bioinformatics and computational biology.

8. Solving the problems of environmental impact on human health and their safety.

9. Earthquake prediction.

10. Geophysical calculations.

11. Astrophysics.

If we ignore a specific area of applications, then the tasks can be divided by two important indicators of working with memory - by the spatio-temporal localization of these calls. Good localization in space is determined by a small spread of addresses of sequentially executed memory accesses and their predictability. Good localization in time - the frequency of calls to the same addresses. 


\section{Result of Testing Modern Systems Using Apex-map}

Moving to the engineering level, we can say that for tasks with good spatio-temporal work with memory, cache memory and schemes for automatically pre-loading data into the cache memory can be effectively used. This can significantly reduce the average memory access time of several hundred processor cycles to fractions of a processor cycle. Such tasks are usually called computational or cachefriendly (cach-friendly) - CF tasks. On tasks with poor spatio-temporal work with memory, the cache memory is useless, so each memory access is hundreds of processor cycles, the processor is idle because of this, and therefore the real performance is in units or even a fraction of a percent of the peak. Such tasks are historically called tasks with intensive irregular work with memory - DIS-tasks. For a example, the dependence of the number of clock cycles of the Power4 processor per memory access has correlation with the spatio-temporal localization, which is artificially changed by the special APEX-MAP test. It can be seen that the best and worst case options differ by two orders of magnitude in terms of execution time [5].

For modern commercially available hardware indicators are approximately the same. However, there are also advances in reducing the average memory access time by using multi-socket boards with large shared memory and a large number of cores that can work with it (see basic performance estimates in Table 1). The average time per memory access is really reduced. This effect is called achieving tolerance of working with memory by entering the simultaneous execution of multiple calls to it due to the large bandwidth of the memory subsystem. This is achieved by generating a large number of accesses of multiple processor cores. Such an effect is also possible for vector processors and processors with massive multi-thread architecture [6].

Table 1. Basic Performance Estimates

\begin{tabular}{|c|c|c|c|c|}
\hline \multirow{2}{*}{ System characteristics } & \multicolumn{4}{|c|}{ Years of implementation } \\
\hline & 2009 & 2011 & 2015 & 2018-2019 \\
\hline Total peak performance & $2 \mathrm{PF}$ & $20 \mathrm{PF}$ & $100-200 \mathrm{PF}$ & $1 \mathrm{EF}$ \\
\hline RAM per computer node & $0.3 \mathrm{~PB}$ & $1 \mathrm{~PB}$ & $5 \mathrm{~PB}$ & $10 \mathrm{~PB}$ \\
\hline Peak performance & $125 \mathrm{GF}$ & $200 \mathrm{GF}$ & $400 \mathrm{GF}$ & $1-10 \mathrm{TF}$ \\
\hline Memory bandwidth & $25 \mathrm{~GB} / \mathrm{s}$ & $40 \mathrm{~GB} / \mathrm{s}$ & $100 \mathrm{~GB} / \mathrm{s}$ & $200-400 \mathrm{~GB} / \mathrm{s}$ \\
\hline Number of processors cores per one computer node & 12 & 32 & $0(100)$ & $0(1000)$ \\
\hline network interface bandwidth per computer node & $1.5 \mathrm{~GB} / \mathrm{s}$ & $10 \mathrm{~GB} / \mathrm{s}$ & $25 \mathrm{~GB} / \mathrm{s}$ & $50 \mathrm{~GB} / \mathrm{s}$ \\
\hline Total number of cores & 225,000 & 3 Million & 50 Million & 0 (Billion) \\
\hline Power consumption & $6 \mathrm{MW}$ & $\sim 10 \mathrm{MW}$ & $\sim 10 \mathrm{MW}$ & $\sim 20 \mathrm{MW}$ \\
\hline Total RAM & $15 \mathrm{~PB}$ & $30 \mathrm{~PB}$ & $150 \mathrm{~PB}$ & $300 \mathrm{~PB}$ \\
\hline I / O Bandwidth & $0.2 \mathrm{~TB} / \mathrm{s}$ & $2 \mathrm{~TB} / \mathrm{s}$ & $10 \mathrm{~TB} / \mathrm{s}$ & $20 \mathrm{~TB} / \mathrm{s}$ \\
\hline
\end{tabular}

The given examples of basic performance estimates with spatially-temporal parameters, memory and real characteristics of equipment operation in such different modes are given in order to illustrate that in practice different types of supercomputers are needed, for example, for $\mathrm{CF}$ and DIS-tasks. As a matter of fact, the structure of modern computer centers usually includes supercomputers of one and the other type. In the vast majority of cases, these are supercomputers for CF-tasks.

The first attempts to select standard equipment based on commercially available components for DIS-tasks related to the introduction of clusters consisting of multi-socket computing nodes with large shared memory on the node. Such cluster fragments are noticeable in the composition of the supercomputer resources of various centers considered below.

Attempts to combine the effective solution of CF- and DIS-tasks in one supercomputer are carried out in projects for promising supercomputers, in particular, under the DARPA HPCS program. The first examples of supercomputers that appeared as a result of work on this program were Cray XE6 and BlueGene / Q.

In these supercomputers, communication networks and the memory subsystem are implemented in a special way to work with the memory of computing nodes through a single address space and at the same time ensure tolerance to large delays in accessing it [7-10].

When considering the supercomputer resources of various US computing centers, it is clear that such supercomputers are actively being introduced and the share of their productivity is usually not less than half of the center's resource. The other half is usually cluster supercomputers.

Such supercomputers and cluster supercomputers, among which there are already fragments for solving CF- and DFtype tasks, or clusters completely specialized for solving such problems, form the basis of the fleet of scientific and technical computer-computer centers of various departments. In the United States, for example, departments are distinguished: the Department of Energy (nuclear weapons and research facilities), the Department of Defense, the National Aerospace Agency, and the National Science Foundation with many universities. All departments combine their supercomputer resources with special networks that are protected to a greater or lesser extent from information attacks. It is also important that the supercomputer centers of these networks, in terms of procurement and operation of equipment, and settlements for supercomputers, work within the framework of a single departmental program.

The presence in almost every center of a supercomputer 
that allows working with large volumes and data streams with increased efficiency suggests that, in addition to applied tasks, they most likely solve log-file analysis tasks in order to control the operation of supercomputers and detect information attacks $[11,12]$.

\section{Discussion of Massive Multi-thread Systems}

In the United States, according to my information analysis, this is the Cray XMT-3 supercomputer for the new NSA center in Utah, and in China, CT-2 (Thunder Clap) supercomputer, created for China's military intelligence. Such supercomputers are the most secret output of work under the DARPA HPCS program and the corresponding Chinese project on high-performance supercomputers within the framework of the 863 program for the development of the main high-tech areas.

These supercomputers will also have unique capabilities for protecting objects from attacks, because by virtue of their features they can effectively analyze large amounts of information from sensors built into the protected object. They will also be an attack weapon due to the same information processing capabilities.

Earlier, characteristics in the form of APEX surfaces of modern commercially available equipment were used. "Slides" are easily visible in different directions when changing the spatio-temporal localization of memory accesses. In the ideal case, they should not be, the surface should be as low as possible and as flat as possible. What characteristics should be expected from new samples of Cray XE6, BlueGene / Q and, finally, from Cray XMT-3 and CT-2? Such information, even according to the commissioned Cray XE6 and BlueGene / Q, has not yet been found, let alone the secret Cray XMT-3 and CT-2 [13, 14].

\section{Conclusions}

Appliance of supercomputers with a new architecture requires certain knowledge and skills. It is being explored now at leading centers. The CASS-MT Center of the Northwest Pacific National Laboratory (PNNL) of the US Department of Energy stands out among the massive multithread architectures, where the possibilities of massive multithread architectures are investigated and software is being developed for them. In the financing and organization of work, the US intelligence community is involved. The project involved PNNL, Cray, Sandia Lab and Georgia University of Technology.

Sandia Lab and Cray have entered into a special cooperation agreement to create data understanding systems and work with large volumes of data. Cray has formed a new division to work in this area [15].

The University of California San-Diego University Center is the leading supercomputer for large data in the United States Science Foundation's XSEDE university program. To solve this problem, the forces of Western Europe are also joining. For example, in the Swiss CSCS center, on the initiative of the Americans from Cray, three types of supercomputers (SGI Altix UV, Cray XMT2 and SX-9) are installed within the framework of the European EISA program, each of which accordingly provides memory latency tolerance, respectively - cache hierarchy - memory, massive multi-thread and vector architecture. The Center carries out various tasks on these supercomputers. According to reports, this center also has a unit composed of US intelligence personnel and operating as a large center for the collection and processing of intelligence information.

\section{References}

[1] J. D. Howard, T. Longstaff. A Common Language for Computer Security Incidents, SANDIA Report 2008-120 p.

[2] Computer Network Security and Privacy Protection, Home Land Security Report, Feb. 19, 2010, 20 p.

[3] Defending Computer Networks against Attack, S \& TR, Cyber Security, Feb. 2011, 17 p.

[4] K. Scanfoe, K. Masone, T. Grance. Computer Security Incident Handling Guide, Recommendations of the NIST, June 2011,50 p.

[5] A. Sharma, J. Barlow, R. Iyer. Analysis of security Data from a Large Computing Organisation, University of Urbana Champaign, March 2011, 14 p.

[6] A. Sharma A. Sharma, J. Barlow. Analysis of Credential Stealing Attacks in an Open Networked Environment, University of Urbana Champaign, April 2011, 16 p.

[7] Trader T. STARnet Alliance Seeks Revolution in Chip Design. HPCWire, January 23, 2013.

[8] Filippov T. V. et al. $20 \mathrm{GHz}$ operation of an asynchronous wave-pipelined RSFQ arithmetic-logic unit. Physics Procedia 36, 2012, pp. 59-65.

[9] Herr A. Rapid Single Flux Quantum Logic. Northrop Grumman. March 2012. 23 slides.

[10] Herr A. Y. et al. An 8-bit carry look-ahead adder with 150ps latency and sub-microwatt power dissipation at $10 \mathrm{GHz}$. arXiv: 1212.2994v1 [quant-ph] Dec 2012. 6 pp.

[11] Molyakov, A. S. New Multilevel Architecture of Secured Supercomputers / A. S. Molyakov // Current Trends in Computer Sciences \& Applications 1 (3) - 2019. - PP. 57-59. - ISSN: 2643-6744 - https://lupinepublishers.com/computerscience-journal/special-issue/CTCSA.MS.ID.000112.pdf. DOI: 10.32474/CTCSA.2019.01.000112.

[12] Molyakov, A. S. Technological Methods Analysis in the Field of Exaflops Supercomputers Development Approaching / A. S. Molyakov, L. K. Eisymont // Global Journal of Computer Science and Technology: Information \& Technology. - 2017. - № 1 (17). - PP. 37-44.

[13] Molyakov, A. S. A Prototype Computer with Non-von Neumann Architecture Based on Strategic Domestic J7 Microprocessor / A. S. Molyakov // Automatic Control and Computer Sciences. - 2016. - № 50 (8). - PP. 682-686. 
[14] Molyakov, A. S. Token Scanning as a New Scientific Approach in the Creation of Protected Systems: A New Generation OS MICROTEK / A. S. Molyakov // Automatic Control and Computer Sciences. - 2016. - № 50 (8). - PP. 687-692.
[15] Molyakov, A. S. Model of hidden IT security threats in the cloud computing environment / A. S. Molyakov, V. S. Zaborovsky, A. A. Lukashin // Automatic Control and Computer Sciences. - 2015. - № 49 (8). - PP. 741-744. 